\title{
Dosimetric study for cervix carcinoma treatment using intensity modulated radiation therapy (IMRT) compensation based on 3D intracavitary brachytherapy technique
}

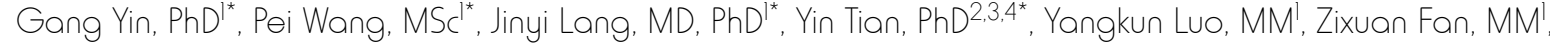 \\ Kin Yip Tam, D Phil, Prof. 5 \\ *These authors contributed equally to this work. \\ 'Department of Radiotherapy, Sichuan Cancer Hospital \& Institute, Chengdu, ${ }^{2}$ Bio-information College, Chongaing University of Posts \\ and Telecommunications, Chongging, ${ }^{3}$ Chongging Key Laboratory of Photoelectronic Information Sensing and Transmiting Technology. \\ Chongaing, ${ }^{4}$ Chongaing High School Innovation Team of Architecture and Core Technologies of Smart Medical System, Chongaing. \\ ${ }^{5}$ Faculty of Health Sciences, University of Macau, Taipa, Macau, China
}

\begin{abstract}
Purpose: Intensity modulated radiation therapy (IMRT) compensation based on 3D high-dose-rate (HDR) intracavitary brachytherapy (ICBT) boost technique (ICBT + IMRT) has been used in our hospital for advanced cervix carcinoma patients. The purpose of this study was to compare the dosimetric results of the four different boost techniques (the conventional 2D HDR intracavitary brachytherapy [CICBT], 3D optimized HDR intracavitary brachytherapy [OICBT], and IMRT-alone with the applicator in situ).

Material and methods: For 30 patients with locally advanced cervical carcinoma, after the completion of external beam radiotherapy (EBRT) for whole pelvic irradiation $45 \mathrm{~Gy} / 25$ fractions, five fractions of ICBT + IMRT boost with $6 \mathrm{~Gy} /$ fractions for high risk clinical target volume (HRCTV), and $5 \mathrm{~Gy} /$ fractions for intermediate risk clinical target volume (IRCTV) were applied. Computed tomography (CT) and magnetic resonance imaging (MRI) scans were acquired using an in situ CT/MRI-compatible applicator. The gross tumor volume (GTV), the high/intermediate-risk clinical target volume (HRCTV/IRCTV), bladder, rectum, and sigmoid were contoured by CT scans.

Results: For ICBT + IMRT plan, values of $\mathrm{D}_{90}, \mathrm{D}_{100}$ of HRCTV, $\mathrm{D}_{90}, \mathrm{D}_{100}$, and $\mathrm{V}_{100}$ of IRCTV significantly increased $(p<0.05)$ in comparison to OICBT and CICBT. The $\mathrm{D}_{2 c c}$ values for bladder, rectum, and sigmoid were significantly lower than that of CICBT and IMRT alone. In all patients, the mean rectum $V_{60}$ Gy values generated from ICBT + IMRT and OICBT techniques were very similar but for bladder and sigmoid, the $\mathrm{V}_{60}$ Gy values generated from ICBT + IMRT were higher than that of OICBT. For the ICBT + IMRT plan, the standard deviations (SD) of $\mathrm{D}_{90}$ and $\mathrm{D}_{2 \mathrm{cc}}$ were found to be lower than other three treatment plans.

Conclusions: The ICBT + IMRT technique not only provides good target coverage but also maintains low doses $\left(D_{2 c c}\right)$ to the OAR. ICBT + IMRT is an optional technique to boost parametrial region or tumor of large size and irregular shape when intracavitary/interstitial brachytherapy cannot be used.
\end{abstract}

Key words: brachytherapy, cervical cancer, cervix carcinoma, dosimetry, IMRT.

\section{Purpose}

Cervix carcinoma is one of the most common malignancies. It is the fourth most common cancer that causes death in women [1]. Cervix carcinoma screening, prevention, and early detection are receiving significant attention. However, a great number of patients are diagnosed with cervical carcinoma when they are already in the advanced stage of the disease, whereas the treatment options are limited to radiation therapy and cisplatin-based chemotherapy.
External beam radiotherapy (EBRT) plus intracavitary brachytherapy are the standard treatments for cervix carcinoma. Clinical treatment found that for tumor of large volume/size or irregular and/or eccentric tumors, the recurrence rate is relatively high $[2,3,4,5,6,7]$. The conventional high-dose-rate (HDR) intracavitary brachytherapy (ICBT) uses point $A$ (defined at $2 \mathrm{~cm}$ superior to the cervical os, and $2 \mathrm{~cm}$ lateral to the tandem) to estimate the dose. However, on many occasions, there is slight 
connection between point $\mathrm{A}$ and the target tumor tissue. The estimated dose, does not necessary reflect the actual radiation dose in the target and OAR, especially for patients with parametrial extension, extensive paravaginal, or distal vaginal involvement, which were unlikely to be encompassed sufficiently by intracavitary application. This discrepancy could be due to the presence of uterine tilt, cervical bias, tumor eccentric, and excessive tumor volume, etc.

Recently, image-guided 3D brachytherapy technique has been widely used in brachytherapy $[8,9,10,11]$. The advantages of 3D intracavitary brachytherapy technique are the possibilities to conform the dose given by brachytherapy to the anatomy of each target volume, at the same time, taking into account both the tumor regression and the position of nearby organs at risk (OAR) [12]. In particular, this technique enables the adjustments of the source dwell time and source position to cover the target volume with the required radiation dose, and maintains the dose of OAR at a relatively reasonable range, resulting in reduction of the normal organ toxicity $[13,14]$. For irregular and/or eccentric tumors or lateral extension to pelvic walls, the adjusting range of the source dwell time and source position are relatively limited for ICBT, which may not effectively guarantee the target dose coverage and control dose in OAR [15]. Tumors with distal parametrial involvement at diagnosis, insufficient response, and/or unfavorable topography after radiochemotherapy represent a therapeutic challenge [16]. In these cases, it may be difficult to devise sufficient dose coverage for target volume using ICBT only. Currently, this problem is being addressed in two different ways, namely, parametrial boost or interstitial implants brachytherapy (ISBT) $[3,16,17]$. For parametrial boost, its effectiveness is poorly documented

Table 1. The features of patients

\begin{tabular}{|c|c|c|}
\hline Content & Number & Percentage (\%) \\
\hline \multicolumn{3}{|l|}{ FIGO stage } \\
\hline IIIA & 13 & 43.3 \\
\hline IIIB & 17 & 56.7 \\
\hline \multicolumn{3}{|l|}{ Age (median age: 43) } \\
\hline$<43$ & 6 & 20 \\
\hline$\geq 43$ & 24 & 80 \\
\hline \multicolumn{3}{|l|}{ Pathological type } \\
\hline Squamous & 29 & 97 \\
\hline Adenocarcinoma & 1 & 3 \\
\hline \multicolumn{3}{|l|}{ Lesion involvement } \\
\hline $\begin{array}{l}\text { Unilateral parametrial } \\
\text { involvement }\end{array}$ & 12 & 40 \\
\hline $\begin{array}{l}\text { Bilateral parametrial } \\
\text { involvement }\end{array}$ & 18 & 60 \\
\hline $\begin{array}{l}\text { Paravaginal or distalvaginal } \\
\text { involvement }\end{array}$ & 13 & 43 \\
\hline
\end{tabular}

FIGO - Federation Internationale de Gynecologie et d'Obstetrique
[18], and there is no strong evidence to validate its routine use. Interstitial brachytherapy is helpful in patients with bulky or an oblite rated endocervical canal, vaginal spread disease. The ISBT technique can be used in combination with ICBT by placing needles in the parametrial region or in large tumor. Intracavitary (IC)-interstitial (IS) BT can achieve good target coverage and is currently recommended as a standard $[15,19,20]$. On some occasions, even with IC-IS BT, the target dose may be insufficient for tumors of large size or challenging topography. A study by Assenholt et al. [16] showed that applicator guided intensity modulated radiation therapy (IMRT) boost in combination with brachytherapy can be used for tumors that are extended over the reach of IC-IS BT applicators.

With the aid of IMRT, it is possible to deliver complex dose distributions for target volumes, and facilitate rapid dose fall-off outside the target volume. Hence, IMRT sometimes can be considered as an option for patients with cervix carcinoma that are unsuitable for ICBT [21]. Recently, dosimetric intercomparisons between brachytherapy and IMRT have been undertaken on carcinoma, prostate cancer, and endometrial cancer radiotherapy $[22,23,24,25,26]$. Some researchers indicated that for conventional HDR brachytherapy with concomitant complementary IMRT boost, it is dosimetrically feasible to improve cervical tumor dose coverage $[2,3,16]$. We note that early work by Duan et al. and Marianne et al. $[2,3]$ have evaluated the combined use of brachytherapy and IMRT technique using a small number of patients for dosimetric studies. However, dosimetric and clinical feasibility of this technique on a larger patient group with parametrial involvement or large tumor have yet to be demonstrated.

In this work, we study the ICBT + IMRT technique to boost the radiation dose for the treatment of patients with cervix carcinoma with the aim to improve target dose coverage without compromising the OARs. A dosimetric comparison was made between ICBT + IMRT and other three simulation boost plans, namely CICBT, OICBT, and IMRT-alone.

\section{Material and methods \\ Patients}

General information: From January 2011 to December 201230 locally advanced cervix carcinoma patients who were diagnosed with parametrial, paravaginal or distal vaginal involvement were treated in our hospital. Patients' ages : $30-65$ years (median age: 43 years); stages: III A of 13 cases, III B of 17 patients (according to 2009 FIGO stage); tumor volumes: GTV $10.4 \mathrm{cc} \pm 7.6 \mathrm{cc}$, HRCTV $43.8 \mathrm{cc} \pm 21.1 \mathrm{cc}$, IRCTV $115.8 \mathrm{cc} \pm 37.9$ cc (Table 1 ).

The study was approved by the Ethics committee of Sichuan Cancer Hospital \& Institute, Chengdu, China. All patients provided written informed consent.

\section{Treatment}

1. All patients underwent EBRT $45 \mathrm{~Gy} / 25$ fractions to the entire pelvis using an IMRT technique with CT-based treatment planning. 
2. Concurrent chemotherapy: During radiotherapy, cisplatin $\left(25 \mathrm{mg} / \mathrm{m}^{2}\right)$ or cisplatin-paclitaxel $\left(135 \mathrm{mg} / \mathrm{m}^{2}\right.$, D1 + DDP $25 \mathrm{mg} / \mathrm{m}^{2}$ ) was administered to patients.

3. Boosting techniques: 3D computed tomography (CT) (in every fraction) and magnetic resonance imaging (MRI) (the first and third and/or fifth fraction) scans were acquired with a Fletcher CT/MRI applicator (Elekta AB, Stockholm, Sweden) in situ. The applicator was subsequently fixed to a board, which can slide between the mobile bed, treatment couch, and computed tomography (CT) couch. A vacuum bag and a thermoplastic mask were used to fix the patient position. In order to reduce the variation of organ in CT/MRI scan, a bladder catheter was used to control bladder filling using a protocol as follows: Before CT/MRI scan, an empty bladder was filled with 100 200 cc physiologic saline according to patient's feels. The same bladder filling protocol was applied before the ICBT delivery in order to acquire similar bladder preparations during treatment and CT/MRI scan. The applicator was reconstructed on the CT images. According to GECESTRO recommendations, gross tumor volume (GTV), HRCTV, and IRCTV were identified from the fusion image of CT and MRI. OAR includes the rectum, bladder, and sigmoid $[27,28]$. The total accumulated dose of EBRT and brachytherapy boost were evaluated in terms of equivalent dose in 2 Gy per fraction (EQD2), using $\alpha / \beta=3$ Gy for OAR and $\alpha / \beta=10$ Gy for targets. The treatment planning aimed to achieve $D_{90}>86 \mathrm{~Gy}$ for HRCTV and $D_{90}>75$ Gy for IRCTV from EBRT and ICBT + IMRT boost. Dose volume constraints for accumulated dose (whole pelvic EBRT and boost) to the OAR were $\mathrm{D}_{2 c c}<90 \mathrm{~Gy}$ for the bladder, and $\mathrm{D}_{2 \mathrm{cc}}<75 \mathrm{~Gy}$ EQD2 for rectum and sigmoid. For every fraction, physical dose volume constrains for OAR can be calculated by following equation [29]:

$$
E Q D 2=n d\left(1+\frac{d}{\alpha / \beta}\right) /\left(1+\frac{2}{\alpha / \beta}\right)
$$

where $n$ is the number of fraction and $d$ is the physical dose per fraction. The calculated physical dose constraints $D_{2 c c}$ OAR were 5.4 Gy per fraction for bladder, and 4.2 Gy per fraction for rectum and sigmoid. In clinical practice, in order to ensure the safety of treatment, we limit the max physical dose $\mathrm{D}_{\max }<5$ Gy for bladder and $\mathrm{D}_{\max }<4.2 \mathrm{~Gy}$ for rectum and sigmoid per fraction.

Total dose optimization included two steps: 1. ICBT dose optimization - the source's dwell time were graphically and manually adjusted using the Oncentra Brachy V4.3 treatment planning system (Elekta AB, Stockholm, Sweden). In order to allow the additional dose contribution from the IMRT boost, the ICBT plan turns the 4 Gy isodose curve away from rectum and sigmoid, and the 4.5 Gy isodose curve away from bladder in every fraction; 2. IMRT plan optimization; a margin of $3 \mathrm{~mm}$ was added to the HRCTV and IRCTV to take into account the uncertainties in the setup of IMRT delivery (defined HRCTVpv, IRCTV-pv). Because of the dose constraints of OAR, some parts of HRCTV or IRCTV could be below the prescription dose in ICBT plan. A seven fields (gantry angle equal distribution) IMRT plan was devised to compensate the area of under prescription dose using an inverse dose optimization tool Oncentra External Beam V4.3 treatment planning system (Elekta AB, Stockholm, Sweden). Intensity modulated radiation therapy plan was optimized on top of ICBT dose using dose volume histograms (DVHs) constraints on the total dose of ICBT and IMRT. The DVH constraints (physical dose) of IMRT optimization in single fraction were as follows: HRCTV-pv, $\mathrm{D}_{90}=6 \mathrm{~Gy}$; IRCTVpv, $\mathrm{D}_{90}=5 \mathrm{~Gy}$; maximum dose of the bladder < 5 Gy; maximum dose of the rectum and sigmoid $<4.2 \mathrm{~Gy}$. In order to improve the feasibility in a clinical workflow, a IMRT plan template has been generated with a standard seven equally spaced beams and a dose constraint library $\left(\mathrm{D}_{90}=6 \mathrm{~Gy}\right.$ for HRCTV-pv; $\mathrm{D}_{90}=5 \mathrm{~Gy}$ for IRCTV-pv; $\mathrm{D}_{\max }<5$ Gy for bladder, and $\mathrm{D}_{\max }<4.2 \mathrm{~Gy}$ for rectum and sigmoid).

In every fraction, once the oncologist has completed the delineation of the target and OAR volumes, the planning of ICBT + IMRT was taken place, which was controlled to be complete within $6 \sim 10$ minutes. Then the ICBT plan was executed on remote after-loading platform within a few minutes to 10 minutes according to the source activity (radioactive sources using ${ }^{192} \mathrm{Ir}$, MicroSelectron automated remote after-loading platform). After the ICBT treatment finished, the patient was transferred to an accelerator (Synergy, Elekta AB, Stockholm, Sweden) with the applicator still in situ. The IMRT execution was guided by the applicator position on kilovoltage cone-beam CT (CBCT), and delivered at a dose rate of 600 $\mathrm{MU} / \mathrm{min}$. Typically, the IMRT plans required a beam-on time of $4 \sim 6$ minutes. Figure 1 demonstrates the whole technological flow of ICBT + IMRT for one fraction.

\section{Simulations of the three dose boosting techniques (CICBT, OICBT, and IMRT-alone)}

1. CICBT: the intracavitary applicator was reconstructed on the CT images. All source positions were of equal interval, and source's dwell times were alike. Reference dose (i.e. dose of point A) was normalized to 6 Gy per fraction (physical dose).

2. OICBT: the intracavitary applicator was reconstructed on the CT images. The source positions and dwell time were optimized manually to achieve $\mathrm{D}_{90}=6 \mathrm{~Gy}$ (physical dose) for HRCTV while maintaining the dose to the OAR as low as possible single fraction.

3. IMRT-alone: the external dose, restriction of DVH, irradiation energy, and gantry angle were the same as IMRT plan optimization of the ICBT + IMRT method.

All boost plan were evaluated to determine the targets dose coverage and the OAR doses. $D_{100}, D_{90}$, and $V_{100}$ were used for targets comparison, while $\mathrm{D}_{2 \mathrm{cc}}$ and $\mathrm{V}_{60} \mathrm{~Gy}$ were considered for OAR. $V_{60}$ Gy represents the volume irradiated to more than 60 Gy (EQD2) by the accumulated dose from EBRT and boost. The boost plans physical isodose level was 2.65 Gy per fraction, since 45 Gy plus $5 \times 2.65$ Gy corresponds to 60 Gy in EQD2 dose. Dose volume histograms cutoff points described before for targets and OAR were compared among the ICBT + IMRT and other three boost plans to evaluate the dosimetric characteristics of ICBT + IMRT technique. 

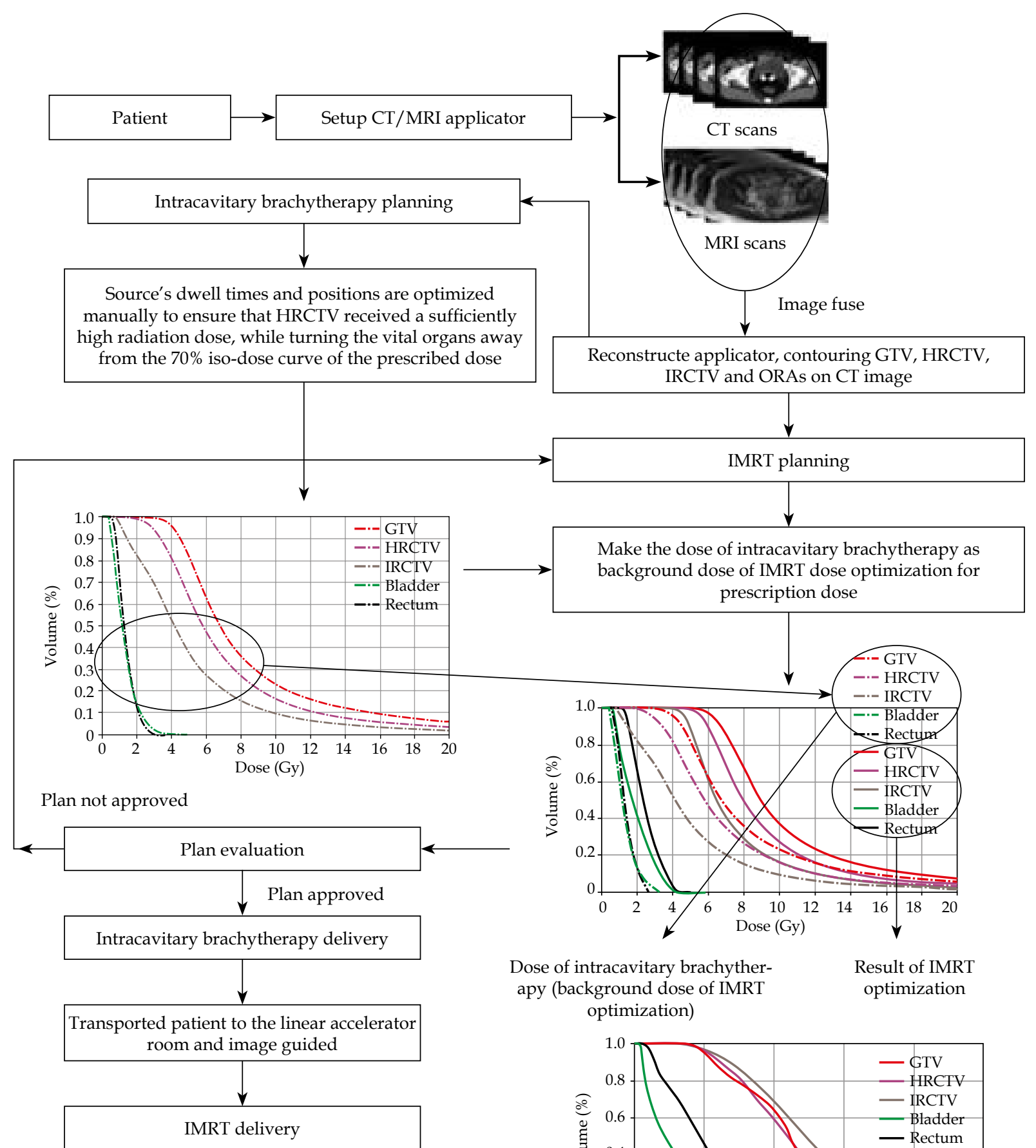

Fig. 1. The whole technological flow of ICBT + IMRT technique

\section{Statistical methods}

Assessment of the four different boost plans for each patient's DVH were accomplished by using paired $t$-test method with the significance level set at $p<0.05$ (two tails). G* power (version 3.1) was used to calculat-

Dose of intracavitary brachytherapy (background dose of IMRT optimization)

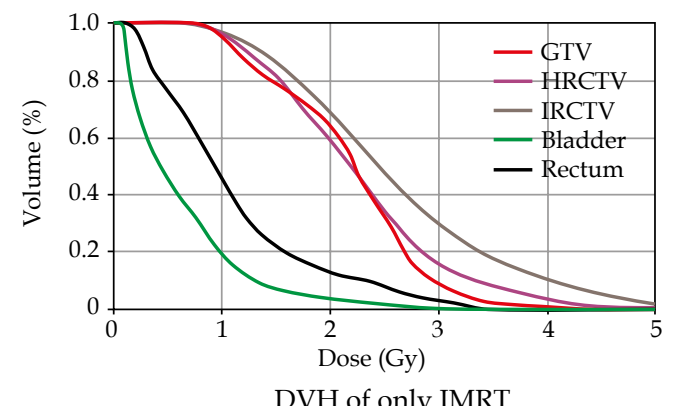

DVH of only IMRT ed the sample size (http://www.softpedia.com/get/ Science-CAD/G-Power.shtml). Parameters were: effect size $\mathrm{dz}=0.7$; type I error probability $(\alpha)=0.05$; type II error probability $(\beta)=0.05$; power $(1-\beta)=0.95$; paired $t$-test significance level set at $p<0.05$ (two tails). The total sample size was found to be about 29 , which is 

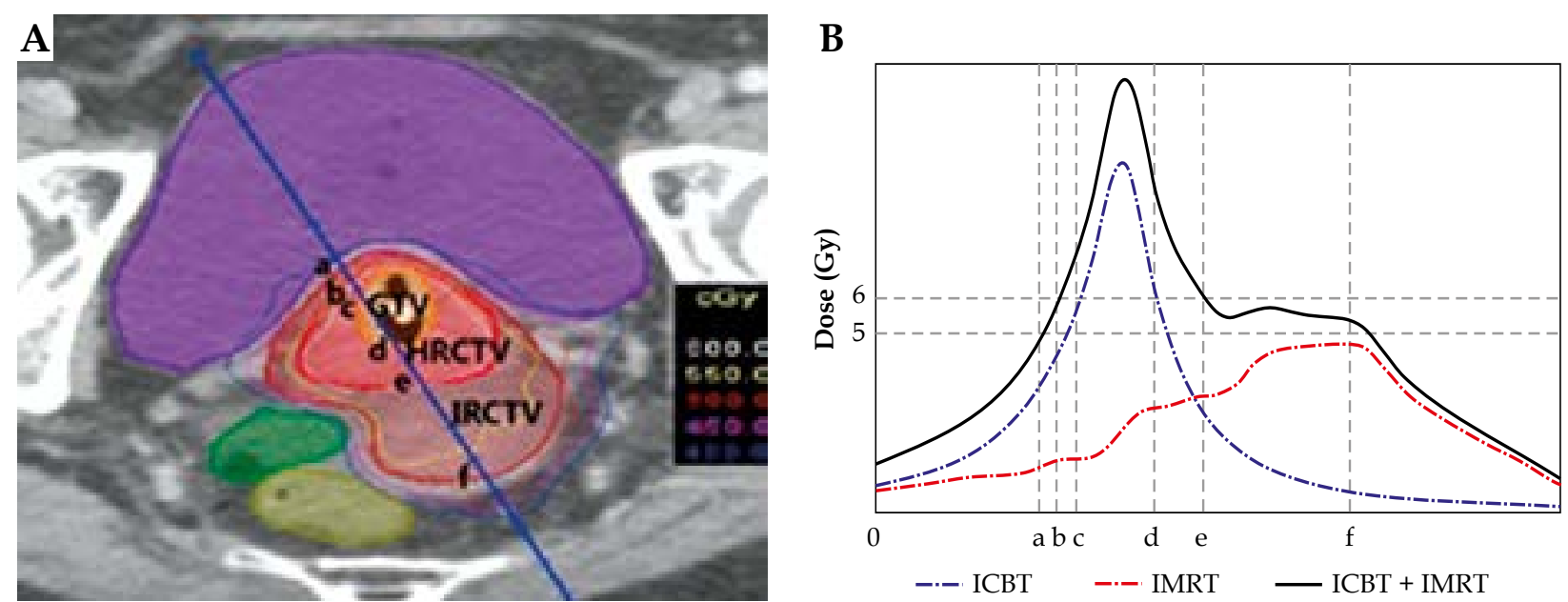

Fig. 2. Dose distributions from ICBT + IMRT plan. A) CT image of a patient. B) Dose distribution corresponds to oblique line of

(A) with, ' $a$ ' to ' $f$ ' for IRCTV range, ' $b$ ' to 'e' for HRCTV range and ' $c$ ' to ' $d$ ' are in the range of GTV (see text for details)

consistent with the number of patients studied in this investigation.

\section{Results}

To illustrate the dose distribution of ICBT + IMRT plan, one patient's axial CT image and corresponding contours (GTV, HRCTV, and IRCTV) as well as the isodose lines are depicted in Figure 2A. Figure 2B shows the dose distribution profiles according to the oblique line in Figure 2A, in which the dash-and-dot line, dotted line, and solid line denote, respectively, ICBT (dose contri- bution of ICBT), IMRT (dose contribution of EBRT), and ICBT + IMRT (all dose contribution of ICBT and IMRT). As shown in Figure 2B, the brachytherapy dose covers most of the GTV volume, whereas the dose of IMRT covers most of the IRCTV volume.

\section{Radiation dose at the tumor target volume and $O A R$}

Table 2 shows the means and standard deviations (SD) of dose parameters $\mathrm{D}_{90}, \mathrm{D}_{100}$, and $\mathrm{V}_{100}$ of target volumes (GTV, HRCTV-pv and IRCTV-pv) for the 30 patients as

Table 2. The mean value and standard deviation of cumulative biological equivalent dose EQD2 ( $D_{90}$ and $\mathrm{D}_{100}$ ) and $\mathrm{V}_{100}$ at the GTV, HRCTV-pv, and IRCTV-pv of the 30 patients

\begin{tabular}{|c|c|c|c|c|c|}
\hline \multirow[t]{2}{*}{ Parameter } & & \multicolumn{4}{|c|}{ Mean \pm SD $($ simple size $=30 ; d f=29)$} \\
\hline & & ICBT + IMRT & CICBT & IMRT-alone & OICBT \\
\hline \multirow[t]{4}{*}{ GTV } & $D_{90}(G y)$ & $103.5 \pm 8.0$ & $174.8 \pm 52.8$ & $88 \pm 0.5$ & $123 \pm 23.3$ \\
\hline & $t ; p$ value & - & $t=7.3 ; p=0.00^{\star}$ & $t=10.5 ; p=0.00^{*}$ & $t=4.4 ; p=0.00^{*}$ \\
\hline & $D_{100}(G y)$ & $84.7 \pm 5.0$ & $104.8 \pm 27$ & $81 \pm 2.3$ & $83.4 \pm 4.9$ \\
\hline & $t ; p$ value & - & $t=4.4 ; p=0.00^{*}$ & $t=5.5 ; p=0.00^{\star}$ & $t=0.7 ; p=0.45$ \\
\hline \multirow[t]{6}{*}{ IRCTV-pv } & $\mathrm{D}_{90}(\mathrm{~Gy})$ & $77.7 \pm 0.9$ & $65.6 \pm 9.1$ & $78.1 \pm 0.8$ & $58.7 \pm 4.0$ \\
\hline & $t ; p$ value & - & $t=7.1 ; p=0.00^{*}$ & $t=1.5 ; p=0.14$ & $t=23.4 ; p=0.00^{*}$ \\
\hline & $D_{100}(G y)$ & $63.7 \pm 2.4$ & $53.3 \pm 3.3$ & $65.1 \pm 2.7$ & $50.4 \pm 1.8$ \\
\hline & $t ; p$ value & - & $t=14.7 ; p=0.00^{*}$ & $t=2.4 ; p=0.02^{*}$ & $t=25 ; p=0.00^{\star}$ \\
\hline & $V_{100}(\%)$ & $94.2 \pm 1.9$ & $74.1 \pm 13.1$ & $93.1 \pm 2.5$ & $60.5 \pm 10.8$ \\
\hline & $t ; p$ value & - & $t=11.0 ; p=0.00^{*}$ & $t=2.6 ; p=0.00^{*}$ & $t=22.7 ; p=0.00^{*}$ \\
\hline \multirow[t]{6}{*}{ HRCTV-pv } & $\mathrm{D}_{90}(\mathrm{~Gy})$ & $88.3 \pm 1.8$ & $105.9 \pm 24.5$ & $85.3 \pm 0.9$ & $85.5 \pm 6.1$ \\
\hline & $t ; p$ value & - & $t=4.0 ; p=0.00^{*}$ & $t=7.8 ; p=0.00^{*}$ & $t=2.5 ; p=0.02^{*}$ \\
\hline & $\mathrm{D}_{100}$ (Gy) & $69.6 \pm 6.6$ & $63.9 \pm 10.6$ & $72.8 \pm 2.0$ & $62.1 \pm 6.6$ \\
\hline & $t ; p$ value & - & $t=2.8 ; p=0.01^{\star}$ & $t=1.2 ; p=0.23$ & $t=9.3 ; p=0.00^{*}$ \\
\hline & $V_{100}(\%)$ & $94.4 \pm 2.8$ & $93.5 \pm 10.3$ & $92.1 \pm 1.6$ & $89.4 \pm 8.7$ \\
\hline & $t ; p$ value & - & $t=0.6 ; p=0.55$ & $t=5.5 ; p=0.00^{*}$ & $t=4.1 ; p=0.00^{*}$ \\
\hline
\end{tabular}

${ }^{*}$ Represents the level of statistical significance $p<0.05$ (two tails).

ICBT - intracavitary brachytherapy, IMRT - intensity modulated radiation therapy, CICBT - 2D HDR intracavitary brachytherapy, OICBT - 3D optimized HDR intracavitary brachytherapy, GTV - gross tumor volume, IRCTV - intermediate risk clinical target volume, HRCTV - high risk clinical target volume, $D_{100}, D_{90}-$ the minimum dose to $100 \%, 90 \%$ of the CTV, $V_{100}$-the percent volume of the post-implant prostate receiving $100 \%$ of the prescribed dose. 
derived from the four boosting techniques. As shown in Table 2, CICBT and OICBT plans provide a high radiation dose for GTV, with $\mathrm{D}_{90}$ and $\mathrm{D}_{100}$ corresponding to 174.8 $\pm 52.8 \mathrm{~Gy}$ and $123 \pm 23.3 \mathrm{~Gy}$, respectively. It is noted that for ICBT + IMRT technique, the cumulative dose for GTV $\left(\mathrm{D}_{90}=103.5 \pm 8.0 \mathrm{~Gy}\right)$ was considerably lower than those of CICBT and OICBT. However, it is much higher than that derived using IMRT-alone technique $\left(\mathrm{D}_{90}=88 \pm 0.5\right.$ Gy). With regard to HRCT-pv and IRCTV-pv, the ICBT + IMRT technique provided considerably higher values of $\mathrm{D}_{90}, \mathrm{D}_{100}$, and $\mathrm{V}_{100}$ than those of the other three techniques.

For OAR, bladder, rectum, and sigmoid, $\mathrm{D}_{2 \mathrm{cc}}$ doses were lower for ICBT + IMRT as compared with CICBT and IMRT-alone plan. Comparing ICBT + IMRT with OICBT, the $\mathrm{D}_{2 \mathrm{cc}}$ for rectum and sigmoid do not show significant difference. However, it was substantially lower for bladder in the ICBT + IMRT plan as compared with OICBT plan. For IMRT-alone, the $\mathrm{V}_{60}$ Gy of OAR were higher than other three plan. $\mathrm{V}_{60}$ Gy for rectum in ICBT + IMRT plan was almost the same as in OICBT plan but it was substantially higher for bladder and sigmoid in the ICBT + IMRT plan as compared with the OICBT plan. The dose parameter $\mathrm{D}_{2 \mathrm{cc}}$ and $\mathrm{V}_{60}$ Gy of bladder, rectum, and sigmoid are showed in Table 3.

\section{Relationships between doses and tumor/normal organ volumes}

The anatomy variations (i.e. volume of bladder, rectum, sigmoid, and tumor) in different patients and between treatment fractions are likely to introduce uncertainty in the dose estimations. In this section, we evaluated the relationships between the dose and the volume of tumor and OAR from the four different boost tech- niques. Figure 3 shows the relationship between average dose $\mathrm{D}_{2 \mathrm{cc}}$ and the average volume of bladder and rectum. Using the CICBT and OICBT techniques, $\mathrm{D}_{2 c c}$ of bladder and rectum show large variations in different bladder and rectum volumes. Generally, a linear relationship can be seen between the doses and the bladder volumes (see the trend lines in Figure 3), suggesting the larger bladder and rectum volume, the higher the $\mathrm{D}_{2 \mathrm{cc}}$ for bladder and rectum. In marked contrast, the doses variation derived using the ICBT + IMRT and IMRT-alone techniques are considerably lower than that of the CICBT and OICBT techniques.

Figure 4 depicts the relationships between the $D_{90}$ of HRCTV, IRCTV, and OAR's $D_{2 \mathrm{cc}}$. For the OICBT and CICBT techniques, no clear relationship between the $\mathrm{D}_{90}$ of HRCTV, IRCTV, and the OAR's $D_{2 c c}$ dose can be seen. On the other hand, the data of the ICBT + IMRT technique appear to cluster at a confined region (see the blue and red symbols in Figure 4).

In order to evaluate the relative dose contribution of ICBT and IMRT using the ICBT + IMRT technique, we calculated the average physical dose contribution of ICBT and IMRT in single fraction, respectively (Figure 5). The relative contribution of ICBT and IMRT are defined by the proportion of the area under the ICBT DVH line (Figure $5 \mathrm{~A}$, gray part) and the area of between ICBT DVH line and total DVH (ICBT + IMRT DVH) line (Figure 5A, brown part). For every single fraction in all patients, the average physical dose contribution of ICBT and IMRT are $90 \pm 0.06 \%$ and $73 \pm 0.05 \%, 55 \pm 0.7 \%$ and $9.6 \pm 0.06 \%, 26.7$ $\pm 0.05 \%$ and $44.8 \pm 0.07 \%$ for GTV, HRCTV-pv, IRCTVpv, respectively. These results suggested that ICBT dose mainly contributed to GTV and HRCTV, while IMRT dose mainly contributed to IRCTV in ICBT + IMRT plan.

Table 3. The mean value and standard deviation of cumulative biological equivalent dose EQD2 of organ at risk (bladder, rectum, sigmoid) $\left(D_{0.1 c c}, D_{1 c c}, D_{2 c c}\right)$ using the four boost techniques

\begin{tabular}{|c|c|c|c|c|c|}
\hline \multirow[t]{2}{*}{ Parameter } & & \multicolumn{4}{|c|}{ Mean \pm SD $($ simple size $=30 ; d f=29)$} \\
\hline & & ICBT + IMRT & CICBT & IMRT-alone & OICBT \\
\hline \multirow[t]{4}{*}{ Bladder } & $\mathrm{D}_{2 \mathrm{cc}}(\mathrm{Gy})$ & $74.4 \pm 2.7$ & $124.5 \pm 32.7$ & $77.2 \pm 2.1$ & $88.5 \pm 14.4$ \\
\hline & $t ; p$ value & - & $t=8.5 ; p=0.00^{*}$ & $t=5.0 ; p=0.00^{\star}$ & $t=5.5 ; p=0.00^{*}$ \\
\hline & $\mathrm{V}_{60 G y}(\mathrm{cc})$ & $56.8 \pm 16.3$ & $64.0 \pm 26.8$ & $73.0 \pm 20.6$ & $42.1 \pm 14.7$ \\
\hline & $t ; p$ value & - & $t=2.3 ; p=0.03^{\star}$ & $t=4.4 ; p=0.00^{\star}$ & $t=2.5 ; p=0.02^{*}$ \\
\hline \multirow[t]{4}{*}{ Rectum } & $\mathrm{D}_{2 \mathrm{cc}}(\mathrm{Gy})$ & $67.9 \pm 2.3$ & $84.1 \pm 16.6$ & $71.0 \pm 2.0$ & $67.8 \pm 8.0$ \\
\hline & $t ; p$ value & - & $t=5.5 ; p=0.00^{\star}$ & $t=7.9 ; p=0.00^{*}$ & $t=0.7 ; p=0.47$ \\
\hline & $\mathrm{V}_{60 G y}(\mathrm{cc})$ & $16.1 \pm 4.3$ & $19.0 \pm 7.6$ & $18.9 \pm 4.7$ & $14.5 \pm 4.9$ \\
\hline & $t ; p$ value & - & $t=2.1 ; p=0.04^{*}$ & $t=2.4 ; p=0.02^{*}$ & $t=1.92 ; p=0.06$ \\
\hline \multirow[t]{4}{*}{ Sigmoid } & $D_{2 c c}(G y)$ & $68.8 \pm 3.1$ & $78.8 \pm 15.8$ & $71.7 \pm 4.0$ & $66.7 \pm 9.4$ \\
\hline & $t ; p$ value & - & $t=3.7 ; p=0.00^{\star}$ & $t=6.3 ; p=0.00^{*}$ & $t=1.5 ; p=0.2$ \\
\hline & $\mathrm{V}_{60 G \mathrm{y}}(\mathrm{cc})$ & $26.0 \pm 8.5$ & $22.7 \pm 9.0$ & $31.3 \pm 10.5$ & $17.9 \pm 7.3$ \\
\hline & $t ; p$ value & - & $t=2.3 ; p=0.03^{*}$ & $t=5.2 ; p=0.00^{*}$ & $t=5.9 ; p=0.00^{*}$ \\
\hline
\end{tabular}

${ }^{*}$ Represents the level of statistical significance $p<0.05$ (two tails)

ICBT - intracavitary brachytherapy, IMRT - intensity modulated radiation therapy, CICBT - 2D HDR intracavitary brachytherapy, OICBT - 3D optimized HDR intracavitary brachytherapy, $D_{2 c c}$ - minimum dose to the most exposed $2 \mathrm{~cm}^{3}, V 60 \%$ - target volume receiving at least $60 \%$ of prescription dose 
A

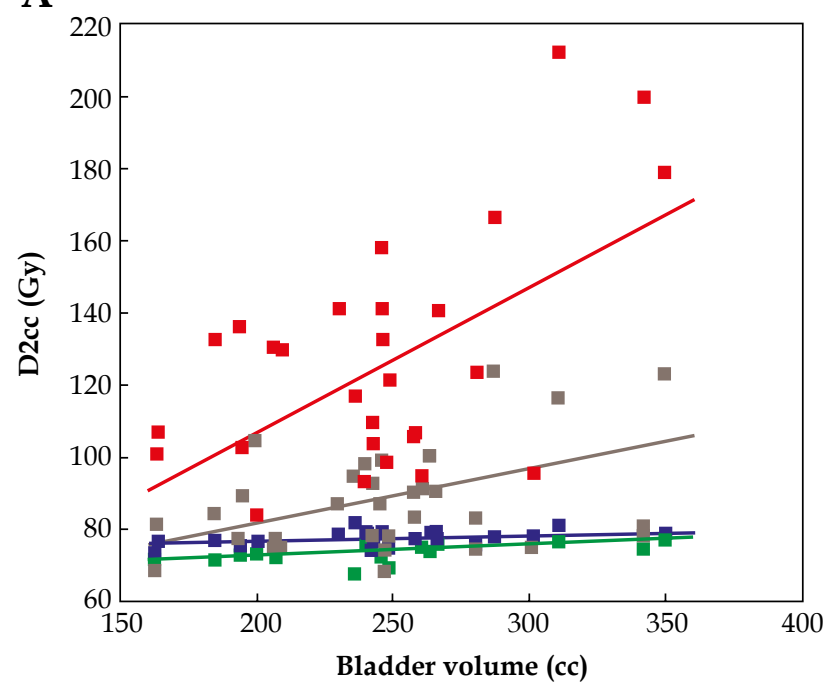

B

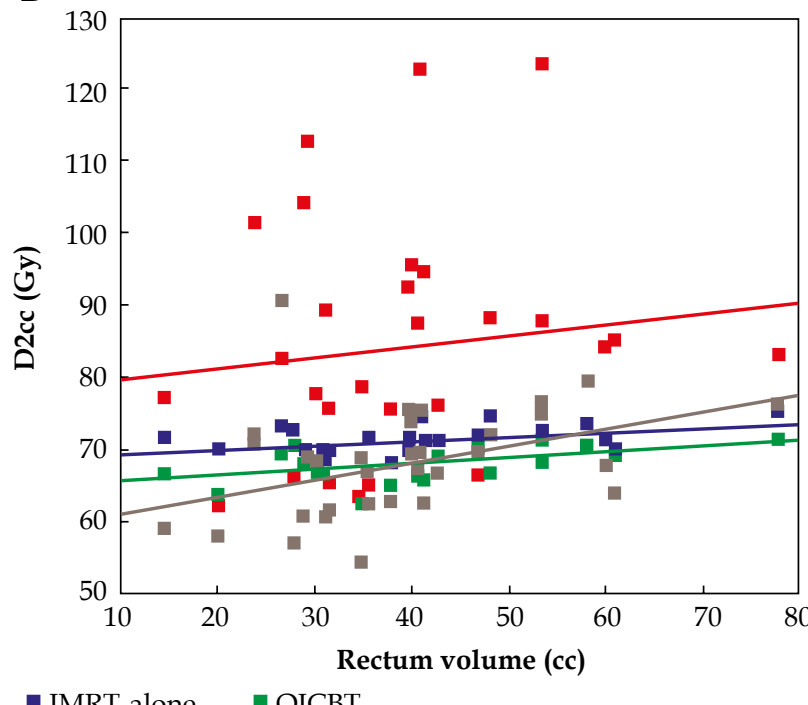

Fig. 3. For four boosting techniques, the relationships between $\mathrm{D}_{2 \mathrm{cc}}$ and volumes of bladder and rectum, respectively
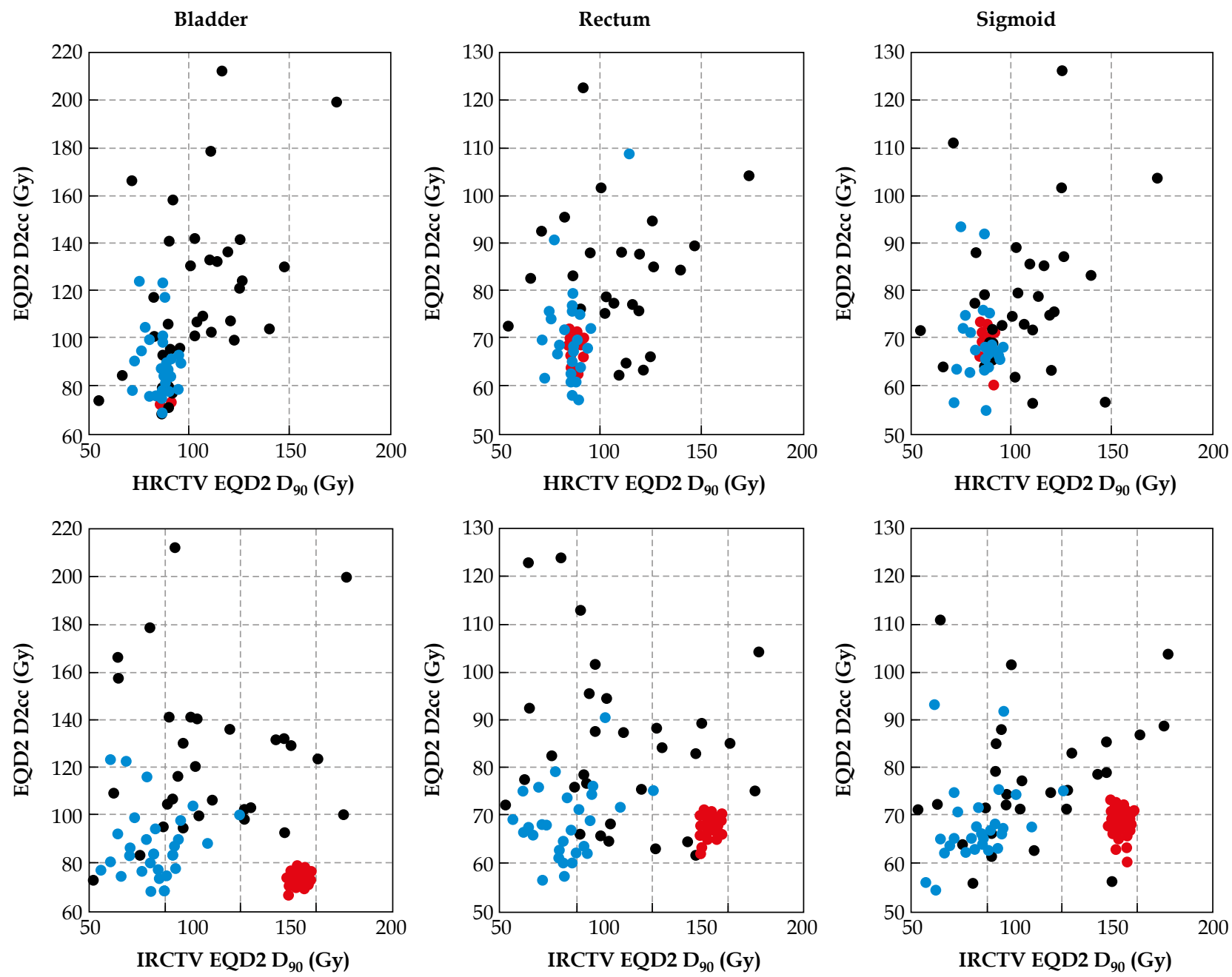

- $\mathrm{ICBT}+\mathrm{IMRT} \quad \bullet \mathrm{CICBT} \quad \bullet \mathrm{OICBT}$

Fig. 4. Scatter plots of the cumulative EQD2 $\mathrm{D}_{90}$ of HRCTV (top) and IRCTV (down) against the three organs at risk (bladder, rectum, and sigmoid) $2 \mathrm{cc}$ cumulative EQD2 $\mathrm{D}_{2 \mathrm{cc}}$ for three boost technique. The scatter points of IMRT-alone are very close to that of ICBT + IMRT and are excluded 
A

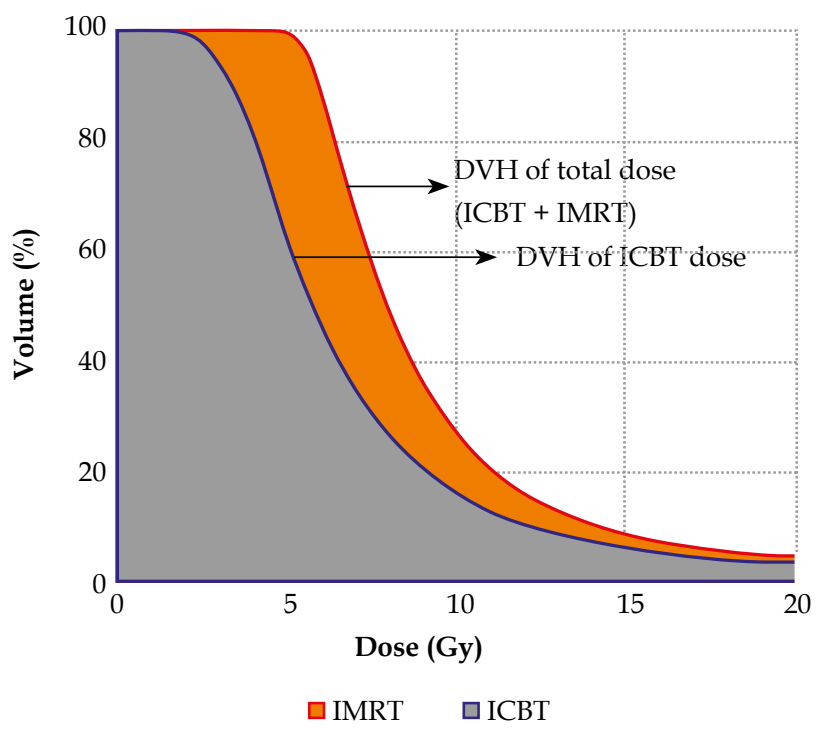

B

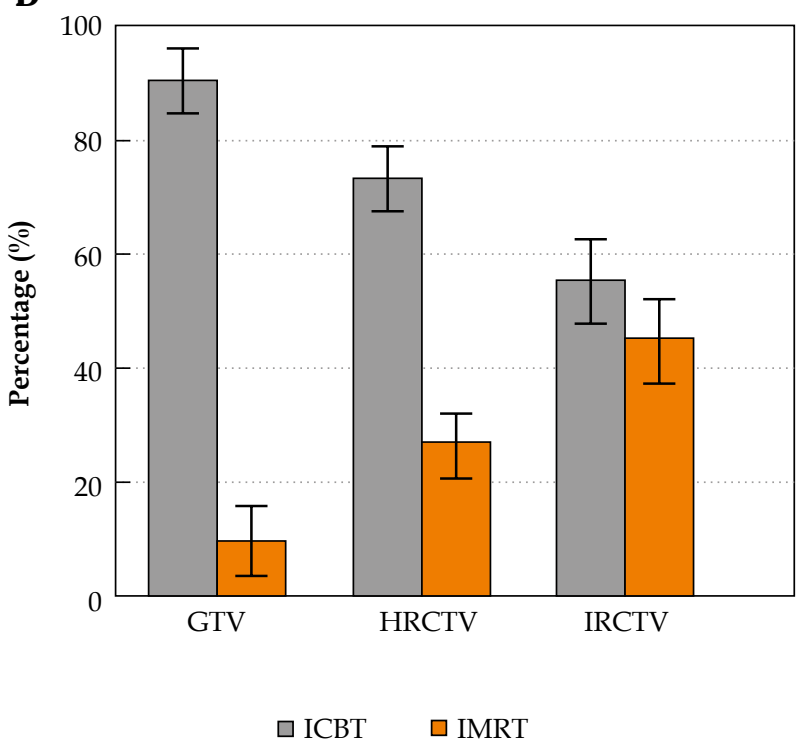

Fig. 5. Dose contributions of ICBT part and IMRT part for the ICBT + IMRT technique. A) Example of HRCTV: the DVH of ICBT dose and ICBT + IMRT dose in one fraction, the area under the DVH line was defined as dose contribution, gray --- ICBT dose contribution; brown --- IMRT dose contribution; the DVH of ICBT + IMRT is the dose contributions from ICBT part plus IMRT part (i.e. gray area plus brown shadow). B) The average dose contribution percentage of ICBT dose and IMRT dose for the target volume GTV, HRCTV, and IRCTV in single fraction

\section{Discussion}

Brachytherapy plays an important role in the cervical cancer radiation therapy. The clinical significance of the technique has been demonstrated in long-term studies [30]. When dealing with parametrial region involvement or large tumor, it is unlikely to be encompassed sufficiently by ICBT only. Because of the close proximity between OAR and target, it is very easy to give excessive dose to normal tissue but inadequate dose for tumor tissue. In this study, we demonstrated that it is technically possible to address this issue using ICBT + IMRT technique. ICBT + IMRT improved the dose coverage to the target without compromising the constraints $\left(D_{2 c c}\right)$ for OAR significantly.

\section{Target volume}

Some evidence indicate that DVH coverage parameters correlate with local tumor control in MRI-guided cervix brachytherapy [10,31,32]. Dimopoulos et al. [32] have shown that patients with an HRCTV $D_{90}$ of greater than 87 Gy achieved a local recurrence rate of approximately $4 \%$, comparing with $20 \%$ in patients with an HRCTV $\mathrm{D}_{90}$ of less than $87 \mathrm{~Gy}$. Our results shown that the cumulative dose of HRCTV-pv $\mathrm{D}_{90}$ is $88.3 \pm 1.9$ Gy by using ICBT + IMRT, which is in good agreement with Dimopoulos et al. [32]. For IRCTV-pv, the $\mathrm{D}_{90}, \mathrm{D}_{100}$, and $\mathrm{V}_{100}$ are significantly low in the CICBT and OICBT plans, which are unlikely to be as effective as the radiation dose derived using ICBT + IMRT.

For HRCTV-pv and IRCTV-pv, the dose parameters $\left(\mathrm{D}_{90}, \mathrm{D}_{100}\right.$, and $\left.\mathrm{V}_{100}\right)$ of ICBT + IMRT are approximately the same as IMRT-alone but ICBT + IMRT shows significantly higher $\mathrm{D}_{90}(103.5 \pm 8.0 \mathrm{~Gy})$ than IMRT-alone $(88$ \pm 0.5 Gy) for GTV. As shown in Figure 5, in ICBT + IMRT plan, the ICBT provided above $90 \%$ dose contribution to GTV. These clearly indicated that the Brachy + IMRT technique maintained high dose for GTV. In the present study, we observed that the IMRT technique alone was difficult to create the same high dose to GTV as CICBT or OICBT. This is mainly due to the fact that IMRT-alone provides a relatively homogeneous dose distribution but cannot provide a high dose gradients distribution similar to that of ICBT, in which the dose rapidly increases as the surface of the applicator is approaching [21]. Some early studies have reported that brachytherapy of the primary cervix tumor could be replaced by IMRT, stereotactic boost, or proton boost [33,34]. Recently, Assenholt [16] pointed out that the brachytherapy dose distribution in the target region is fundamentally different from a homogeneous IMRT dose distribution. In brachytherapy, 50\% of the target is typically irradiated to more than $150 \%$ of prescribed dose, which seems to be of the utmost importance for local control. Without the use of brachytherapy, the overall survival decreased by more than $10 \%$ [16]. Tanderup et al. pointed out that brachytherapy is irreplaceable for locally advanced cervical cancer [35]. Meanwhile, many studies $[36,37,38,39,40]$ demonstrated the critical importance of brachytherapy in the treatment of cervical cancer. For ICBT + IMRT technique, the contributions of brachytherapy dose are $90 \pm 0.06 \%, 73 \pm 0.05 \%$ to GTV and HRCTV, respectively. Obviously, brachytherapy still plays a major role in ICBT + IMRT treatment, while IMRT offers additional dose compensation for the underdose areas, such as IRCTV $(44.8 \pm 0.07 \%)$.

The ISBT was reported to be feasible and good target coverage achievable in large tumors $[15,19,20]$. On some 
occasions, even with combined ICBT and ISBT, the dose may be insufficient for some tumors with large residual tumor/or challenging topography, especially for irregular and/or eccentric tumor distribution [16]. In these cases, ICBT + IMRT may be an alternative option.

Parametrial extension is a poor prognostic factor in cervical cancer. Parametrial boost has traditionally been accomplished using direct anterior and posterior fields EBRT boost with midline shielding [39]. However, this technique has several disadvantages. Firstly, tumor coverage may well be compromised. The greater inhomogeneity dose on the target was suboptimal. Although brachytherapy can deliver a high dose to the primary tumor, coverage of the lateral parametrium out to the pelvic side wall is likely to be suboptimal. Secondly, there is a potential for excess toxicity $[41,42,43]$, because of geometric uncertainties during the delivery of midline block plan. Thus, the OAR have a greater risk of falling into irradiation field, resulting in overdose, especially to the areas of adjacent to OAR. Using ICBT + IMRT boost technique, this problem can be resolved because the ICBT and IMRT were planned on same CT image for every fraction, allowing a precise volumetric addition of dose.

\section{Organs at risk}

For the treatment of cervix cancer, the total dose including both EBRT and brachytherapy is the most significant factor affecting the incidence of late adverse side effects $[44,45,46]$. Considering the side effects and toxicity in OAR, some researcher report that the cumulative dose constraints are $\mathrm{D}_{2 \mathrm{cc}}<75$ Gy for the rectum and sigmoid and $\mathrm{D}_{2 \mathrm{cc}}<90 \mathrm{~Gy}$ for the bladder $[47,48]$. Our results showed that the cumulative dose $\mathrm{D}_{2 \mathrm{cc}}$ for rectum, sigmoid, and bladder are 67.9 $\pm 2.3 \mathrm{~Gy}, 68.8 \pm 3.1 \mathrm{~Gy}$, and $74.4 \pm 2.7$ Gy, respectively (see Table 3 ), which are lower than that of published papers $[47,48]$.

Studies conducted by Hashim and Vinod pointed out that the OAR doses assessed by DVH criteria were higher than the ICRU point doses [49,50]. Georg et al. reported that the parameters $D_{2 c c}$ and $D_{1 c c}$ were predictive for rectal toxicity [48]. Dose volume histograms parameters were also found to be good predictors for bladder toxicity [48]. These suggested that OAR doses and the side/toxic effects were generally correlated. However, the clinical impact of increasing the $\mathrm{V}_{60}$ Gy has not yet fully understood $[3,16]$. Prior studies have pointed out that large volume in the pelvic region receiving more than $60 \mathrm{~Gy}$ correlated to side effects [45]. The results also showed that using an applicator guided IMRT boost instead of ICBT will result in a substantially larger $\mathrm{V}_{60} \mathrm{~Gy}[3,16]$. It should be mentioned that ICBT + IMRT plan resulted in a larger OAR's $\mathrm{V}_{60}$ Gy as compared with the OICBT plan, but ICBT + IMRT plan is better than IMRT-alone on $\mathrm{V}_{60}$ Gy because the main dose contribution is coming from ICBT and not from IMRT. By increasing the external beam contribution, $\mathrm{OAR}^{\prime} \mathrm{S} \mathrm{V}_{60}$ Gy value shows substantial increase. Generally, target dose distribution of IMRT is more uniform comparing with brachytherapy. It is remained to be seen whether the differences in dose distributions associated with the $V_{60}$ Gy values would lead to difference side effects. This subject and the effect of low doses to an enlarged volume of normal tissue would deserve further investigation.

\section{Volume/dose relationship for target and OAR}

Although point A dose and target volume for traditional brachytherapy treatment were not directly linked, the target volume and dose were approximately in an inversely proportional relationship [51]. In clinical treatment, the tumor volume of different patients and its spatial distribution may vary significantly. As can be seen in Figure 3, the relationships between the volume and the radiation doses are generally in direct proportion (particularly in CICBT and OICBT techniques). Literature evidence suggested that when the tumor volume is greater than $31 \mathrm{cc}$ and its HRCVT $\mathrm{D}_{90}$ dose is less than the prescribed dose, the tumor will be significantly underdosed in the case of point A plan [52]. The results shown in Figure 4 confirm that with brachytherapy alone (e.g. CICBT or OICBT), little correlation in dose is seen between the tumor target and the normal organ. As for CICBT and OICBT techniques, the tumor target doses (GTV, HRCTVpv, IRCTV-pv) are inversely proportional to the tumor volumes. Nevertheless, CICBT or OICBT techniques, as the tumor volume increases, the risk of target underdose also increases. As can be seen from Figures 4 and 5, where ICBT + IMRT technique can adaptively maintain the prescribed doses for target (HRCTV-pv, IRCTV-pv), which are not affected by volumes of target and normal organs.

\section{Uncertainties in the experimental setup}

It has been pointed out that for brachytherapy the changes in the uterine axis, uterine length, bladder, rectum filling state, and vaginal packing, could result in fluctuations in spatial location $[53,54]$. These motions could potentially affect the accurate delivery of complementary IMRT. In this study, we performed CT and/or MRI scans for patients in every fraction to minimize the inter-fractional motion. During the treatment process, the applicator was fixed inside the patient's body, which played an important role to control the tumor motion. This could minimize the impact on the changes in the physiological condition of the individual patient. Together with external beam radiation image guide system (such as CBCT), the uncertainty of patient position for IMRT delivery can be minimized. Taking into consideration the delivery uncertainty, we incorporated a margin of $3 \mathrm{~mm}$ to the HRCTV and IRCTV for ICBT + IMRT plan. It is noted that a margin of $3 \mathrm{~mm}$ uncertainties in IMRT delivery for target has been adopted by some researchers $[3,16]$. The risk of significant cold and hot spots in the target and OAR were low even if the organs were moved $3 \mathrm{~mm}$ during the treatment [16]. Our study corroborated these findings and demonstrated that the dose distribution of the combined boost plan was stable within uncertainties of $3 \mathrm{~mm}$.

In ICBT + IMRT plan optimization, we used OAR DVH constraints to account for the uncertainty in OARs: $\mathrm{D}_{\max }<86 \mathrm{~Gy}$ for the bladder, and $\mathrm{D}_{\max }<75$ Gy for rectum and sigmoid. These constraints were more severe as 
compared with those adopted in a related study by some researchers $[3,16]$ (i.e. $D_{2 c c}<90$ Gy for the bladder, and $\mathrm{D}_{2 \mathrm{cc}}<75 \mathrm{~Gy}$ or rectum and sigmoid). On the other hand, Joshua Schindel reported that no more than $\pm 1.5 \mathrm{~mm}$ applicator displacements were allowed for both point A and ICBT plans, which can avoid the uncertainties of dose greater than $10 \%$ [42].

\section{Limitations of this study}

Our results showed that ICBT + IMRT plan led to a substantially larger $\mathrm{V}_{60}$ Gy. Although the clinical impact of increased $V_{60}$ Gy volume is currently unknown, previous studies have pointed out that a large volume receiving more than $60 \mathrm{~Gy}$ is correlated with side effects [45]. By increasing the external beam contribution, OAR's $\mathrm{V}_{60}$ Gy value will increase substantially. Hence, it is recommended that ICBT + IMRT technique is only suitable for locally advanced patients with paravaginal involvement or large tumor.

Although the current results of ICBT + IMRT techniques for gynecological applications are encouraging, more detailed studies on practical aspects of precision dose delivery need to be performed. Small geometrical variations with overlap of high dose regions of ICBT and IMRT may lead to dramatic consequences for organs at risk, while cold spots between the ICBT and the IMRT dose contributions have to be avoided. Although some researcher consider that a margin of $3 \mathrm{~mm}$ uncertainties in IMRT delivery for target is safe $[3,16]$, the real geometrical variations between ICBT and IMRT are very difficult to find because ICBT and IMRT plans were executed separately and anatomy variations (i.e. volume of bladder, rectum, sigmoid, and tumor, etc.) in different patients may be a problem. In order to avoid the cold and hot spots as much as possible, we have undertaken the following measures to minimize the setup uncertainties and internal motions: firstly, it was assumed that the target moves together with the applicator, and the applicator was fixed to a board to minimize setup uncertainties. Secondly, a bladder catheter was used to control bladder filling, so that the bladder volume was the same during HDR ICBT and IMRT delivery. Thirdly, the IMRT plan was delivered immediately after the ICBT with the applicator still in situ. The rectum and sigmoid movements were assessed visually on $\mathrm{CBCT}$ in as much detail as the CBCT image quality allowed.

In our study, we tried to compensate for the area of under prescription dose by applying a dose escalation to the part of the target with IMRT but the dose in the central part of the tumor may be different with EBRT and ICBT dose distributions, even with the same coverage and the same dose to the outer part of the tumor. There is no evidence to suggest which dose levels escalation should be performed in order to obtain the same level of local control.

All dose constraints and dose values such as $D_{2 c c}$ so far were based on an external beam dose of 45 Gy to 50 Gy. Because of the contribution of EBRT in ICBT + IMRT plan, the total EBRT dose may be increased to more than $50 \mathrm{~Gy}$. In our study, $\mathrm{D}_{\max }$ could be considered as DVH constraints for OAR. Increasing the external beam contribution parameters like $V_{20}$ or $V_{30}$ or similar, will show substantial increase in OAR dose, so intermediate and low dose constraints such as $\mathrm{V}_{20}$ or $\mathrm{V}_{30}$ should be considered in ICBT + IMRT plan optimization in our future studies. At the moment, there is no evidence to suggest which volume dose constraints level of OAR should be employed to maintain the same level of side effects. Additional confirmatory studies with larger numbers of patients and longer follow-up time are required to evaluate the side effects of low dose radiation to large OAR volume.

\section{Conclusions}

For the patients with large tumor involving parametrial region, the ICBT + IMRT technique not only provides excellent target coverage but also maintains low doses $\left(\mathrm{D}_{2 \mathrm{cc}}\right)$ to the OAR. Combining the advantage of ICBT and IMRT, it was dosimetrically and logistically feasible to apply the ICBT + IMRT in a clinical setting. ICBT + IMRT technique offers an alternative solution for large tumor when intracavitary/interstitial brachytherapy cannot be used.

\section{Acknowledgements}

We thank the support from the Chongqing advanced and applied basic research project \#cstc2015jcyjA10024, the National Nature Science Foundation of China (\#61471075), and the Science and Technology Development Fund, Macao S.A.R. (FDCT) (project reference no. 086/2014/A2).

\section{Disclosure}

Authors report no conflict of interest.

\section{References}

1. Stewart BW, Wild CP (eds.). World Cancer Report 2014. World Health Organization, Geneva 2014; Chapter 5.12.

2. Duan J, Kim RY, Elassal S et al. Conventional high-doserate brachytherapy with concomitant complementary IMRT boost: a novel approach for improving cervical tumor dose coverage. Int J Radiat Oncol Biol Phys 2008; 71: 765-771.

3. Assenholt MS, Petersen JB, Nielsen SK et al. K. A dose planning study on applicator guided stereotactic IMRT boost in combination with 3D MRI based brachytherapy in locally advanced cervical cancer. Acta Oncol 2008; 47: 1337-1343.

4. Briot E, Crevoisier D, Petrow P et al. Dose-volume-histogram analysis for tumor and critical organs in intracavitary brachytherapy of cervical cancer with the use of MRI. Radiother Oncol 2001; 60 (Suppl 1): S3.

5. Fellner C, Pötter R, Knocke TH et al. Comparison of radiography- and computed tomography-based treatment planning in cervix cancer in brachytherapy with specific attention to some quality assurance aspects. Radiother Oncol 2001; 58: 53-62.

6. Schoeppel SL, Ellis JH, LaVigne ML et al. Magnetic resonance imaging during intracavitary gynecologic brachytherapy. Int J Radiat Oncol Biol Phys 1992; 23: 169-174.

7. Gerstner N, Wachter S, Reinstadler E et al. Radiography compared to sectional image (CT/MR)-based 3D treatment planning in cervix cancer brachytherapy: results of a perspective comparative trial. Radiother Oncol 2001; 60 (Suppl 1): S3. 
8. Lindegaard JC, Fokdal LU, Nielsen SK et al. MRI-guided adaptive radiotherapy in locally advanced cervical cancer from a Nordic perspective. Acta Oncol 2013; 52: 1510-1519.

9. Nomden CN, de Leeuw AAC, Roesink JM et al. Clinical outcome and dosimetric parameters of chemo-radiation including MRI guided adaptive brachytherapy with tandem-ovoid applicators for cervical cancer patients: a single institution experience. Radiother Oncol 2013; 107: 69-74.

10. Pötter R, Dimopoulos J, Georg P et al. Clinical impact of MRI assisted dose volume adaptation and dose escalation in brachytherapy of locally advanced cervix cancer. Radiother Oncol 2007; 83: 148-155.

11. Mazeron R, Gilmore J, Dumas I et al. Adaptive 3D image-guided brachytherapy: a strong argument in the debate on systematic radical hysterectomy for locally advanced cervical cancer. Oncologist 2013; 18: 415-422.

12. Petric P, Mohammed-Al-Hammadi N. MRI findings at image guided adaptive cervix cancer brachytherapy: radiation oncologist's perspective. J Contemp Brachytherapy 2014; 6: 215222.

13. Lindegaard JC, Tanderup K, Nielsen SK et al. MRI-guided 3D optimization significantly improves DVH parameters of pulsed-dose-rate brachytherapy in locally advanced cervical cancer. Int J Radiat Oncol Biol Phys 2008; 71: 756-764.

14. De Brabandere M, Mousa AG, Nulens A et al. Potential of dose optimization in MRI-based PDR brachytherapy of cervix carcinoma. Radiother Oncol 2008; 88: 217-226.

15. Dimopoulos JC, Kirisits C, Petric P et al. The Vienna applicator for combined intracavitary and interstitial brachytherapy of cervical cancer: clinical feasibility and preliminary results. Int J Radiat Oncol Biol Phys 2006; 66: 83-90.

16. Assenholt MS, Vestergaard A, Kallehauge JF et al. Proof of principle: applicator-guided stereotactic IMRT boost in combination with 3D MRI-based brachytherapy in locally advanced cervical cancer. Brachytherapy 2014; 13: 361-368.

17. Viswanathan AN, Creutzberg CL, Craighead P et al. International brachytherapy practice patterns: a survey of the Gynecologic Cancer Intergroup (GCIG). Int J Radiat Oncol Biol Phys 2012; 82: 250-255.

18. Fenkell L, Assenholt M, Nielsen SK et al. Parametrial boost using midline shielding results in an unpredictable dose to tumor and organs at risk in combined external beam radiotherapy and brachytherapy for locally advanced cervical cancer. Int J Radiat Oncol Biol Phys 2011; 79: 1572-1579.

19. Fokdal L, Tanderup K, Hokland SB et al. Clinical feasibility of combined intracavitary/interstitial brachytherapy in locally advanced cervical cancer employing MRI with a tan$\mathrm{dem} /$ ring applicator in situ and virtual preplanning of the interstitial component. Radiother Oncol 2013; 107: 63-68.

20. Kirisits C, Lang S, Dimopoulos J et al. The Vienna applicator for combined intracavitary and interstitial brachytherapy of cervical cancer: Design, application, treatment planning, and dosimetric results. Int J Radiat Oncol Biol Phys 2006; 65: 624630.

21. Shwetha B, Ravikumar M, Siddanna R et al. Dosimetric comparison of high dose rate brachytherapy and intensity-modulated radiation therapy for cervical carcinoma. Med Phys 2011; 36: 111-116.

22. Low DA, Grigsby PW, Dempsey JF et al. Applicator-guided intensity-modulated radiation therapy. Int J Radiat Oncol Biol Phys 2004; 52: 1400-1406.

23. Malhotra HK, Avadhani JS, deBoer SF et al. Duplicating a tandem and ovoids distribution with IMRT: a feasibility study. J Appl Clin Med Phys 2007; 8: 9-17.

24. Wahab SH, Malyapa RS, Mutic $S$ et al. Treatment planning study comparing HDR and AGIMRT for cervical cancer. Med Phys 2004; 31: 734-743.
25. Hermesse J, Biver S, Jansen $\mathrm{N}$ et al. A dosimetric intercomparison of HDR brachytherapy, IMRT, and Helical Tomotherapy in prostate cancer radiotherapy. Strahlenther Onkol 2009; 185: 736-742.

26. Aydogan B, Mundt AJ, Smith BD et al. A dosimetric analysis of intensity-modulated radiation therapy (IMRT) as an alternative to adjuvant high-dose-rate (HDR) brachytherapy in early endometrial cancer patients. Int J Radiat Oncol Biol Phys 2006; 65: 266-273.

27. Haie-Meder C, Pötter R, Van Limbergen E et al. Recommendations from Gynaecological (GYN) GEC-ESTRO Working Group (I): Concepts and terms in 3D image based 3D treatment planning in cervix cancer brachytherapy with emphasis on MRI assessment of GTV and CTV. Radiother Oncol 2005; 74: 235-245.

28. Pötter R, Haie-Meder C, Van Limbergen E et al. Recommendations from gynaecological (GYN) GEC ESTRO working group (II): Concepts and terms in 3D image-based treatment planning in cervix cancer brachytherapy-3D dose volume parameters and aspects of 3D image-based anatomy, radiation physics, radiobiology. Radiother Oncol 2006; 78: 67-77.

29. De Leeuw AA, Van de Kamer JB, Moerland MA et al. The effect of alternative biological modelling parameters (alpha/ beta and half time of repair T (1/2)) on reported EQD2 values in the treatment of advanced cervical cancer. Radiother Oncol 2011; 101: 337-342.

30. Hanks GE, Herring DF, Kramer S. Patterns of care outcome studies: results of the national practice in cancer of the cervix. Cancer 1983; 51: 959-967.

31. Dimopoulos JC, Pötter R, Lang S et al. Dose-effect relationship for local control of cervical cancer by magnetic resonance image-guided brachytherapy. Radiother Oncol 2009; 93: 311-315.

32. Dimopoulos JC, Lang S, Kirisits C et al. Dose-volume histogram parameters and local tumor control in magnetic resonance image-guided cervical cancer brachytherapy. Int $\mathrm{J} \mathrm{Ra}$ diat Oncol Biol Phys 2009; 75: 56-63.

33. Jhingran A. Potential advantages of intensity-modulated radiation therapy in gynecologic malignancies. Semin Radiat Oncol 2006; 16: 144-151.

34. Wolfson AH. Magnetic resonance imaging and positron-emission tomography imaging in the 21st century as tools for the evaluation and management of patients with invasive cervical carcinoma. Semin Radiat Oncol 2006; 16: 186-191.

35. Tanderup K, Eifel P J, Yashar CM et al. Curative radiation therapy for locally advanced cervical cancer: brachytherapy is NOT optional. Int J Radiat Oncol Biol Phys 2014; 88: 537-539.

36. Han K, Milosevic M, Fyles A et al. Trends in the utilization of brachytherapy in cervical cancer in the United States. Int J Radiat Oncol Biol Phys 2013; 87: 111-119.

37. Logsdon MD, Eifel PJ. Figo IIIB squamous cell carcinoma of the cervix: an analysis of prognostic factors emphasizing the balance between external beam and intracavitary radiation therapy. Int J Radiat Oncol Biol Phys 1999; 43: 763-775.

38. Eifel PJ, Moughan J, Erickson B et al. Patterns of radiotherapy practice for patients with carcinoma of the uterine cervix: a patterns of care study. Int J Radiat Oncol Biol Phys 2004; 60: 1144-1153.

39. Good J, Lalondrelle S, Blake P. Point: Parametrial irradiation in locally advanced cervix cancer can be achieved effectively with a variety of external beam techniques. Brachytherapy 2012; 11: 77-79.

40. Thompson SR, Delaney GP, Gabriel GS et al. Patterns of care study of brachytherapy in New South Wales: cervical cancer treatment quality depends on caseload. J Contemp Brachytherapy $2014 ; 6: 28-32$. 
41. Huang EY, Lin $\mathrm{H}, \mathrm{Hsu} \mathrm{HC}$ et al. High external parametrial dose can increase the probability of radiation proctitis in patient with uterine cervical cancer. Gynecol Oncol 2000; 79: 406-410.

42. Schindel J, Zhang W, Bhatia SK et al. Dosimetric impacts of applicator displacements and applicator reconstruction-uncertainties on 3D image-guided brachytherapy for cervical cancer. J Contemp Brachytherapy 2013; 5: 250-257.

43. Mohamed S, Kallehauge J, Fokdal L et al. Parametrial boosting in locally advanced cervical cancer: combined intracavitary/interstitial brachytherapy vs. intracavitary brachytherapy plus external beam radiotherapy. Brachytherapy 2015; 14 : 23-28.

44. Perez CA, Breaux S, Bedwinek JM et al. Radiation therapy alone in the treatment of carcinoma of the uterine cervix. II. Analysis of complications. Cancer 1984; 54: 235-246.

45. Barillot I, Horiot J, Maingon P et al. Impact on treatment outcome and late effects of customized treatment planning in cervix carcinomas: baseline results to compare new strategies. Int J Radiat Oncol Biol Phys 2000; 48: 189-200.

46. Perez CA, Grigsby PW, Lockett MA. Radiation morbidity in carcinoma of the uterine cervix: dosimetric and clinical correlation. Int J Radiat Oncol Biol Phys 1999; 44: 855-866.

47. Conibear J, Lowe G, Hoskin PJ. High-precision MRI-guided adaptive brachytherapy for cervical carcinoma. Int $J$ Hyperthermia 2012; 28: 501-508.

48. Georg P, Lang S, Dimopoulos JC et al. Dose-volume histogram parameters and late side effects in magnetic resonance image-guided adaptive cervical cancer brachytherapy. Int J Radiat Oncol Biol Phys 2011; 79: 356-362.

49. Vinod SK, Caldwell K, Lau A et al. A comparison of ICRU point doses and volumetric doses of organs at risk (OARs) in brachytherapy for cervical cancer. J Med Imaging Radiat Oncol 2011; 55: 304-310.

50. Hashim N, Jamalludin Z, Ung NM et al. CT Based 3-Dimensional Treatment Planning of Intracavitary Brachytherapy for Cancer of the Cervix: Comparison between Dose-Volume Histograms and ICRU Point Doses to the Rectum and Bladder. Asian Pac J Cancer Prev 2014; 15: 5259-5264.

51. Terahara A, Nakano T, Ishikawa A et al. Dose-volume histogram analysis of high dose rate intracavitary brachytherapy for uterine cervix cancer. Int J Radiat Oncol Biol Phys 1996; 35: 549-554.

52. Tanderup K, Nielsen SK, Nyvang GB et al. From point A to the sculpted pear: MR image guidance significantly improves tumour dose and sparing of organs at risk in brachytherapy of cervical cancer. Radiother Oncol 2010; 94: 173-180.

53. Srivastava A, Datta NR. Brachytherapy in cancer cervix: time to move ahead from point A? World J Clin Oncol 2014; 5: 764774.

54. Datta NR, Basu R, Das KJ et al. Problems in reporting doses and volumes during multiple high-dose-rate intracavitary brachytherapy for carcinoma cervix as per ICRU Report 38 : a comparative study using flexible and rigid applicators. Gynecol Oncol 2003; 91: 285-292. 